\title{
DIFFUSE COPLANAR SURFACE BARRIER DISCHARGE PLASMA TREATMENT AS A PART OF TECHNOLOGY FOR MANUFACTURING OF NANOFIBER-BASED FILTERS
}

\author{
${ }^{1}$ Oleksandr GALMIZ, 'Michal FLEISCHER, 'David PAVLIŇÁK, ${ }^{1}$ Jakub KELAR,

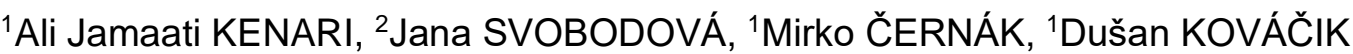 \\ ${ }^{1}$ Department of Physical Electronics, CEPLANT, Masaryk University, Brno, Czech Republic, EU, \\ o.galmiz@mail.muni.cz \\ ${ }^{2}$ NAFIGATE Corporation a.s., Prague, Czech Republic, EU
}

https://doi.org/10.37904/nanocon.2021.4311

\begin{abstract}
Nanofiber membranes are made of synthetic polymers mainly by electrospinning technology. The key point for creating a functional nanofiber membrane for water and air filters is to meet basic key properties such as filtration efficiency, mechanical resistance, and resistance to fouling and chemicals. Design and manufacturing of the advanced nanofiber-based filters urgently require new environment-friendly and cost-effective surface treatments without the use of organic solvents and caustic solutions. To address this need, as an alternative, the atmospheric-pressure plasma treatment offers to be used for surface activation of polymer textile materials serving as a substrate for electrospun nanofiber. Nanofiber carriers represented by polypropylene non-woven were pre-treated by dielectric barrier discharge in continuous mode to improve the adhesion between the produced nanofibers and substrate. The increased adhesive forces to carrier substrate were confirmed by two peeling tests. The fact that the robust and effective atmospheric-pressure diffuse coplanar surface barrier discharge technology, primarily developed and optimized for the plasma treatment of textile and fibrous material, can be easily implemented in the industrial production lines predetermines this technology for in-line a large throughput manufacturing of advanced nanofiber-based filters.
\end{abstract}

Keywords: Nanofibers, plasma, adhesion, spunbond non-woven polypropylene

\section{INTRODUCTION}

Currently, the requirements to achieve filtration efficiency in conjunction with the requirement for the highest possible breathability of filter material force the manufacturer to look for new material combinations and technological processes for the production of filter media. Filter media containing a nanofiber filter membrane have proven to be an advantageous and economical option for capturing submicron particles. Such media can therefore be advantageously used for the production of filters and protective aids with FPP3 filtration efficiency, including protection against viral particles. These nanofiber membranes are made of synthetic polymers mainly by electrospinning technology. This fabrication method which is based on high electrostatic forces to elongation and thinning of flowed polymer fiber is very popular for its simplicity, efficiency, and usability of most industrially expanded polymers.

Such nanofiber properties as great porosity and high surface area make this nanomaterial interesting for applications focusing on filtration, fibrous reinforcements in synthetic or biological composites, electronic devices, or tissue engineering [1]. The nanofibers themselves are tender material and are very sensitive to mechanical manipulation. Moreover, the low adhesion forces of the nanofibers to the untreated carrier make them very complicated material to manipulate with, and a high degree of the nanofibers damage during their 
processing. The filtration efficiency and mechanical resistance are also determined by such parameters as good adhesion of the membrane to the substrate.

At present, many efforts are being addressed to modify the surface properties of polymer fibers to improve the performance of these materials and to pave the way to new technological applications. Nowadays a plasma treatment is a frequently used method for physical and chemical modification of polymer surfaces [2]. Under the bombardment of active species generated by the plasma, the polymer surface can be modified by removing surface contamination, introducing new chemical functional groups, or deposition of thin coating [3]. The diffusivity of the diffuse coplanar surface barrier discharge (DCSBD) plasma and the high efficiency showed to be one of the most efficient in such cases.

In this work, the DCSBD plasma sources operated in the air at atmospheric pressure were used to improve the adhesion properties of the substrate represented by spunbond non-woven polypropylene (SB NWPP) after plasma treatment and the nanofibers' adhesion to the substrate.

\section{EXPERIMENTAL SETUP}

\subsection{Nanofibres deposition}

The spinning of nanofiber mesh was carried out on the NanospiderTM NSLAB 500 machine (Elmarco, Czech Republic). Conditions of electrospinning were as follows: $55 \mathrm{kV}$ applied HV, $140 \mathrm{~mm}$ electrode to collector distance, $6 \mathrm{rpm}$ rotating speed of the working electrode. As the working electrode was used the $10 \mathrm{~cm}$ length rotating wire electrode. The nanofiber mats were collected on spunbond non-woven polypropylene of $17 \mathrm{gsm}$ substrate with a $25 \mathrm{~mm} / \mathrm{min}$ shift speed. The rewinding itself was provided by a self-standing rewinding device, ensuring the correct fabric downforce to the electrodes, the displacement of the carrier during electrospinning, and the speed and feed direction.

Polyvinylidene fluoride (PVDF) was chosen as a starting polymer with well-defined properties for the study of the adhesion of nanofibers to the substrate and their modification in plasma. Electrospinning solution was prepared by dissolving the PVDF powder (purchased by Nafigate a.s., Czech Republic) in a solution (6.75/50 $\mathrm{g} / \mathrm{ml}$ ) of N,N-Dimethylacetamide (anhydrous, $99.8 \%$, Sigma Aldrich). The $1 \%$ of tetraethylammonium bromide (Sigma Aldrich) was added to the final solution to increase the electrical conductivity. After pellets agitation, the solution was tempered with continuous stirring for $1 \mathrm{~h}$ at $40^{\circ} \mathrm{C}$ and later $23 \mathrm{~h}$ at $25^{\circ} \mathrm{C}$.

\subsection{Plasma treatment}

The processing of the carrier fabric was performed using a planar or curved DCSBD (CDCSBD) electrode. Plasma was generated in the form of a surface dielectric barrier discharge in air at atmospheric pressure at a supplied sinusoidal voltage of $15-30 \mathrm{kHz}$ frequency and amplitude of $10 \mathrm{kV}$. The tested power of the DCSBD was in the range $200-600 \mathrm{~W}$.

The main advantage of a concavely curved DCSBD electrode allowing the processing of sensitive materials, as well as various foils and soft fabrics. The process parameters were the same as for the planar electrode. Photos in Figure $1(\mathbf{a}, \mathbf{c})$ shows the DCSBD electrodes prepared for the plasma treatment process.

\subsection{Strike-through time measurements}

The test to determine the liquid penetration time (urine imitation) for nonwovens was performed according to European standard ISO 9073-8. The materials used for this test consist of infiltrating reference pads (filter papers) and urine imitation according to ISO 3696 (a solution with $9 \mathrm{~g} / \mathrm{l}$ chloride sodium in the water of purity 3). An electronic time meter, with the possibility of reading the time up to hundreds of seconds, was used to measure the strike-through time for $5 \mathrm{ml}$ of testing solution. 

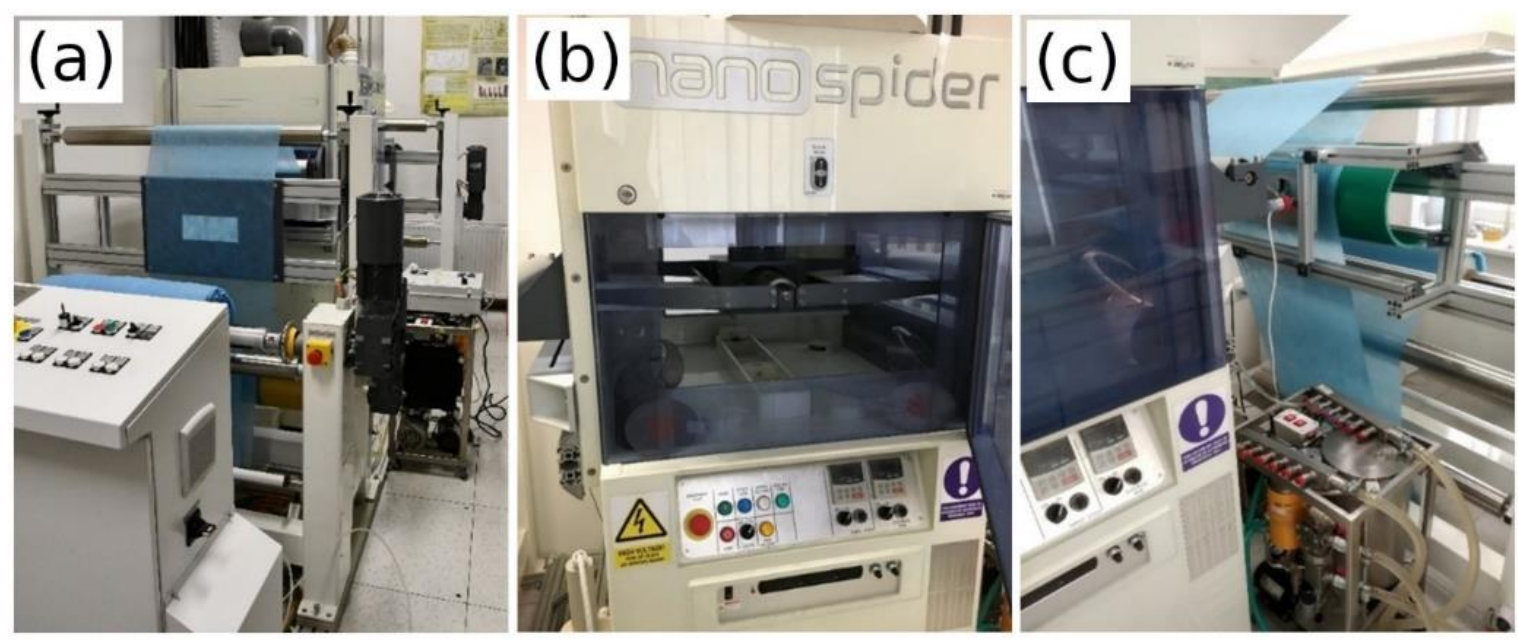

Figure 1 The line for the carrier plasma pre-treatment and the nanofibers production: a) planar DCSBD electrode, b) the Nanospider NSLAB 500 device, c) concavely curved DCSBD electrode

\subsection{Adhesion measurements - Peel test}

Adhesion measurements were performed on a TA.XTplusC Texture Analyzer with a $50 \mathrm{~N}$ load cell at a head speed of $1 \mathrm{~mm} / \mathrm{s}$ from Stable Micro Systems. The force required to detach the calibrated adhesive tape, under 90 degree angle, from the test specimens (peel test) was measured. Reference samples and samples that underwent plasma treatment on DCSBD and CDCSBD systems were tested. A peel test was done immediately after the plasma treatment. Testing was performed on more than 10 samples and the resulting values were obtained from the arithmetic mean of the measured data. Exponent Connect software was used to process the data.

\subsection{Adhesion measurements - Loop test}

Adhesion tests were performed by measuring the force required to tear the deposited nanofiber layer from an area of $25 \mathrm{~mm} \times 25 \mathrm{~mm}$. A TA.XTplusC Texture Analyzer (Stable Micro Systems, UK) with a $5 \mathrm{~N}$ load cell was used for the measurement. A method that was used for these measurements called "loop test" is a modified method based on the method used for adhesion measurement of the adhesive tape.

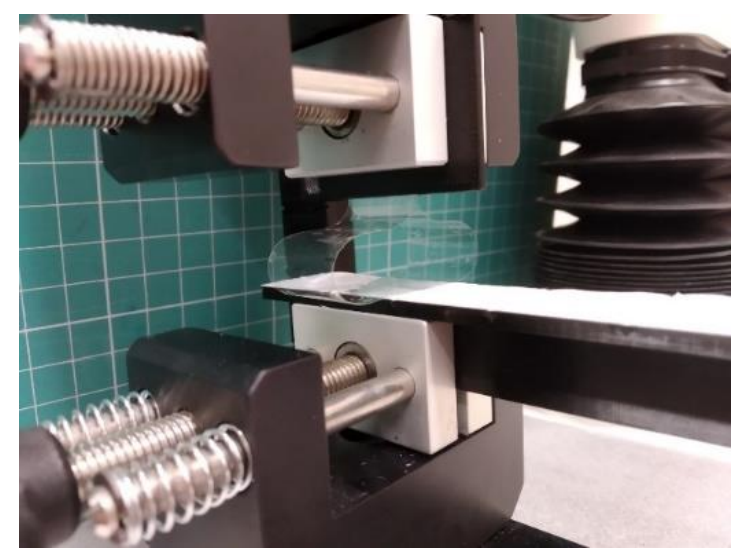

Figure 2 Measurement of the adhesive force between the SB NWPP substrate and the PVDF nanofibers by the loop test method

The sample with cut $25 \mathrm{~mm} \times 25 \mathrm{~mm}$ sections, was glued to a plastic holder with double-sided adhesive tape on the side of the substrate, which was then fixed in the lower jaws of the measuring device. A test adhesive 
tape with sufficiently high adhesion, $10 \mathrm{~cm}$ long, was placed in a controlled manner in the upper movable jaws so as to form a loop (see Figure 2). Keeping the same length $(10 \mathrm{~cm}$ ) and the shape of the loop a reproducible pressure force of the test adhesive tape was achieved. The tape was not pressed to the sample in any other manner. Subsequently, the jaws were moved away, and the magnitude of the applied force vs. jaw position was recorded. The speed of movement of the jaw head was $5 \mathrm{~mm} / \mathrm{s}$. The trigger force was $1 \mathrm{~g}$ (i.e. $10 \mathrm{mN}$ ). As the parameter for comparison, the area under the obtained curve, corresponding to the mechanical work necessary to tear off the layer was calculated and compared. The maximum force and the mean force were then determined from the digital recording of the measurement. The maximum force was always reached just before tearing the nanofiber layer from the textile substrate. In a general minimum of 10 samples were tested for every condition.

\section{RESULTS}

\subsection{Strike-through time measurements}

It was observed that the urine imitation was able to penetrate through the reference SB NWPP substrate sample. However, non-uniform behavior was recorded. On some samples of the non-woven, strike-through time values exceeded 600 seconds. Mainly the average time of penetration was around 16 seconds with a standard deviation of 10 seconds. Even such a short treatment time as 1 second by DCSBD plasma was enough to reduce this time to 2 seconds. The effect of plasma treatment using both DCSBD configurations was comparable and did not exceed 3 seconds even with error ranges.

\subsection{Adhesion measurements - Peel test}

For this experimental setup, both planar and curved configurations of DCSBD were tested. While treating the carrier material both configurations of the electrodes proved to have a desirable effect in adhesion increase for a chosen carrier material. In the case of porous carrier textiles treated in direct contact with the thin plasma layer generated on the ceramic plate of the DCSBD electrode, the surface free energy increases, which also indicates the increase of the adhesion forces. Plasma treatment applying the $400 \mathrm{~W}$ power increases the adhesion force of the SB NWPP material significantly (see Figure 3). The application of the planar electrode configuration resulted in the increase of the adhesion force by $450 \%$. With a low treatment time (up to 3 seconds) the measurements behave in the same manner. No significant tearing and therefore no major force deviations were observed, see Figure 4. Measuring the samples after plasma exposure for 5 seconds and longer, a different behavior was recorded. In most cases, the material still showed results close to the previous and did not have any significant influence on the measured force values. But, in some cases, a complete tearing of the used fabric was observed (Figure 4c). This difference could be explained by the inhomogeneity of the material that also corresponds to the differences in the strike-through time measurements. Some samples were plasma treated at $600 \mathrm{~W}$ of the supplied power. Samples were torn in the same way as after the plasma exposure at $400 \mathrm{~W}$. With the increase of the exposure time more and more fabrics stayed glued to the adhesion tape. The change occurs at an exposure time of $10 \mathrm{~s}$ when tearing becomes more pronounced and the material damage could take place shortly after the beginning of the test.

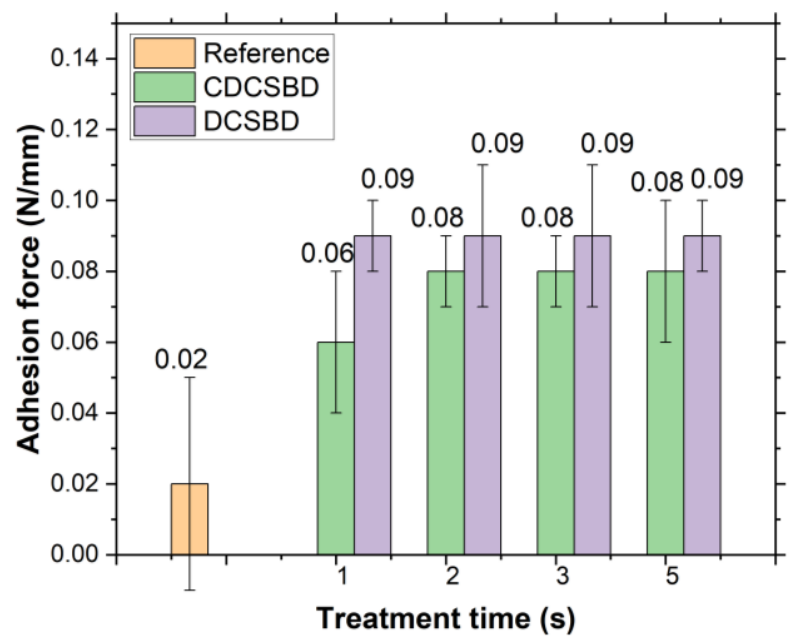

Figure 3 Adhesion force measurements on SB NWPP $17 \mathrm{gsm}$ 


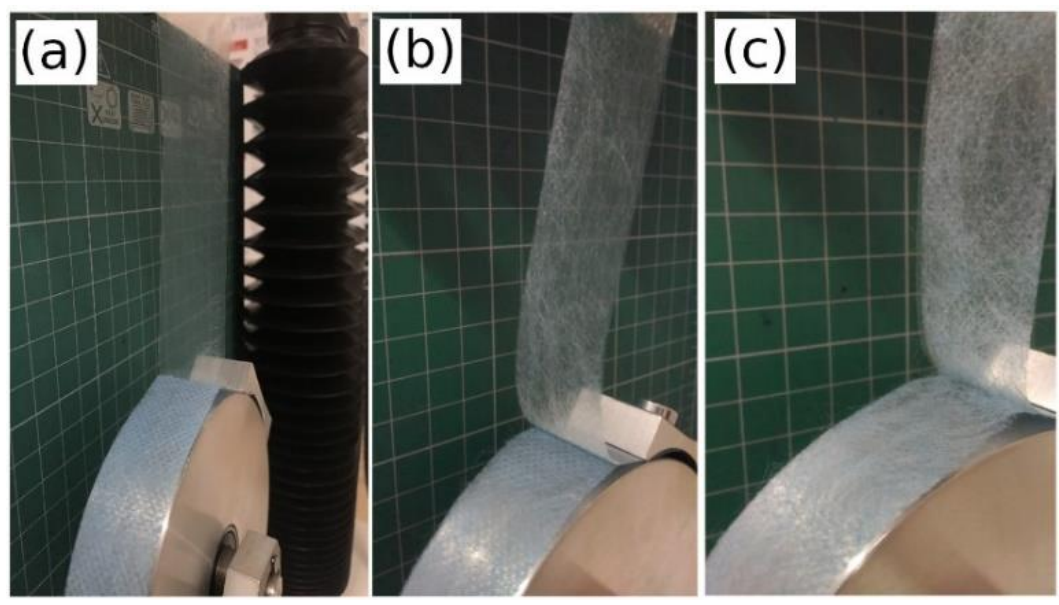

Figure 4 Photo of the a) reference sample SB NWPP after the peel test, samples that have undergone plasma treatment using DCSBD with an exposure time of b) $2 \mathrm{~s}, \mathrm{c}) 5 \mathrm{~s}$

\subsection{Adhesion measurements - Loop test}

When measuring reference samples, the applied nanofiber layer was always completely separated from the textile substrate. The values of adhesion force in most cases were so small that even a very sensitive strain gauge could not observe them. In some cases, a force of $0.01 \mathrm{~N}$ was observed. However, the plasma treatment often increased the adhesion to the substrate. The samples of the carrier substrate were plasma treated with the exposure time of $1,2,5$, and 10 seconds at the supplied power of 400 and 600 Watts. The PVDF nanofibers were deposited on the carrier right after the plasma treatment. The results obtained after the loop test measurements are summarized in Table 1 and Table 2.

Increasing the treatment time as well as supplied power results in the increased value of the work needed for the detachment of fibers from the carrier. Even one second plasma treatment of the carrier gives the opportunity for further material processing. Increasing the exposure time to 10 seconds results in more than $550 \%$ increase of the adhesion force in comparison to the 1 second plasma pre-treatment.

Table 1 The results of the loop test after $400 \mathrm{~W}$ plasma treatment

\begin{tabular}{|c|c|c|c|}
\hline Treatment time (s) & Max (N) & Mean (N) & Area (N*mm) \\
\hline 1 & $0.07 \pm 0.01$ & $0.022 \pm 0.006$ & $0.37 \pm 0.06$ \\
\hline 2 & $0.08 \pm 0.02$ & $0.05 \pm 0.01$ & $0.45 \pm 0.016$ \\
\hline 5 & $0.13 \pm 0.02$ & $0.05 \pm 0.02$ & $0.72 \pm 0.21$ \\
\hline 10 & $0.25 \pm 0.04$ & $0.09 \pm 0.01$ & $2.27 \pm 0.58$ \\
\hline
\end{tabular}

Table 2 The results of the loop test after $600 \mathrm{~W}$ plasma treatment

\begin{tabular}{|c|c|c|c|}
\hline Treatment time (s) & Max (N) & Mean (N) & Area $\left(\mathbf{N}^{*} \mathbf{m m}\right)$ \\
\hline 1 & $0.09 \pm 0.02$ & $0.02 \pm 0.01$ & $0.46 \pm 0.08$ \\
\hline 2 & $0.12 \pm 0.04$ & $0.05 \pm 0.01$ & $0.78 \pm 0.18$ \\
\hline 5 & $0.14 \pm 0.05$ & $0.05 \pm 0.02$ & $0.89 \pm 0.46$ \\
\hline 10 & $0.28 \pm 0.04$ & $0.11 \pm 0.02$ & $2.56 \pm 0.61$ \\
\hline
\end{tabular}

From the results above it is obvious that the adhesion forces of the carrier substrate, as well as the adhesion force between the carrier and the nanofibers, increased significantly after the plasma treatment. 


\section{CONCLUSION}

It was shown that the DCSBD plasma treatment has an outstanding effect on the adhesion of the SB NWPP textile. The strike-through time test showed that even after seconds of plasma treatment the permeability of urine imitation (according to ISO 9073-8) increased significantly. The material became hydrophilic what is a good indicator for the adhesion enhancement. The results of the peel force test indicate that the plasma treatment promotes the adhesion force increase at least 3 times. The dependence of this increase on the exposure time is not reflected in the results of the measured strain gauges, but with the help of photographs, we observe an increased tearing of the fabric with increasing time. A special method for measuring nanofibers' adhesion to the substrate was invented. With the help of this method the samples used for this study were tested. The results show a weak holding of nanofibers on the reference carrier substrate. The manipulation with the untreated carrier of nanofibers is a problem now and requires further processing. Samples that were plasma treated show dependence in increasing adhesion with increasing the exposure time. It was shown that the work needed to detach the nanofibers from the substrate increased significantly.

By testing two configurations of the DCSBD electrode, two applied powers, and several exposure times, it was concluded that the best parameters for treating the SB NWPP substrate without damaging it are planar electrode, $400 \mathrm{~W}$ of supplied power, and ten seconds of exposure time. Applying these parameters, homogeneous plasma treatment was achieved with the reproducible results of increased adhesion force.

\section{ACKNOWLEDGEMENTS}

This work was supported by project In-line plasma technology for a large throughput manufacturing of advanced nanofiber-based filter fabrics (No. E01-14) funded by the European Light Industries Innovation and Technology initiative, which is funded by the European Union COSME Program for Competitiveness of Companies and Small and Medium Enterprises.

\section{REFERENCES}

[1] ŠVACHOVÁ, V.; VOJTOVÁ, L.; PAVLINÁK, D.; VOJTEK, L.; SEDLÁKOVÁ, V.; HYRŠL, P.; ALBERTI, M.; JAROŠ, J.; HAMPL, A.; JANČǍ̌, J. Novel electrospun gelatin/oxycellulose nanofibers as a suitable platform for lung disease modeling. Mater. Sci. Eng. C. [online]. 2016, vol. 67, pp. 493-501. Available from: https://doi.org/10.1016/.j.msec.2016.05.059.

[2] FRIEDRICH, J. The Plasma Chemistry of Polymer Surfaces. Wiley-VCH Verlag GmbH \& Co. KGaA: Weinheim, Germany, 2012. ISBN 9783527648009.

[3] KEDRONOVÁ, E.; ZAJÍČKOVÁ, L.; HEGEMENN, D.; KLÍMA, M.; MICHLíČEK, M.; MANAKHOV, A. Plasma Enhanced CVD of Organosilicon Thin Films on Electrospun Polymer Nanofibers. Plasma Process. Polym. [online]. 2015, vol. 12, pp. 1231-1243. Available from: https://doi.org/10.1002/ppap.201400235. 